\title{
Polysomnographic phenotype as a risk factor for cardiovascular diseases in patients with obstructive sleep apnea syndrome: a retrospective cohort study
}

\author{
Sunmin Park, Beomsu Shin, Ji-Ho Lee, Seok Jeong Lee, Myoung Kyu Lee, Won-Yeon Lee, \\ Suk Joong Yong, Sang-Ha Kim \\ Department of Internal Medicine, Yonsei University Wonju College of Medicine, Wonju, South Korea \\ Contributions: (I) Conception and design: S Park, SH Kim; (II) Administrative support: SH Kim; (III) Provision of study materials or patients: All \\ authors; (IV) Collection and assembly of data: S Park; (V) Data analysis and interpretation: S Park, SH Kim; (VI) Manuscript writing: All authors; (VII) \\ Final approval of manuscript: All authors. \\ Correspondence to: Sang-Ha Kim, MD, PhD. Department of Internal Medicine, Yonsei University Wonju College of Medicine, 20 Ilsanro Wonju, \\ Gangwon 26326, South Korea. Email: sanghakim@yonsei.ac.kr.
}

Background: Obstructive sleep apnea syndrome (OSAS) is associated with the development of
cardiovascular diseases caused by hypoxemia during sleeping. We classified OSAS phenotypes based on
polysomnographic findings and aimed to evaluate that the unique phenotypes would be differentially
associated with risk of cardiovascular disease. Methods: This retrospective and observational study assessed adult patients who underwent polysomnography at the Wonju Severance Christian Hospital from November 2008 to February 2018. The OSAS phenotypes were classified as apnea-predominant, hypopnea-predominant, and respiratory effortrelated arousal (RERA)-predominant based on the polysomnography results. The polysomnographic data were collected and analysed, and clinical features such as medical history and comorbidities were assessed by a review of the electronic medical records.

Results: A total of 860 adult patients were classified as apnea-predominant $(n=220)$, hypopnea-predominant $(\mathrm{n}=119)$, or RERA-predominant $(\mathrm{n}=275)$. The hypopnea-predominant group had significantly higher rates of hyperlipidaemia $(\mathrm{P}<0.001)$, heart failure $(15.5 \%, \mathrm{P}<0.001)$, and coronary artery disease $(20.9 \%, \mathrm{P}=0.005)$ than the other groups. After classifying the patients according to severity of the hypopnea index, logistic regression analyses adjusted for age, sex, and smoking history revealed that the hypopnea index increased the risk for coronary artery disease and heart failure.

Conclusions: The hypopnea-predominant group would be a specific phenotype that has a differential association with the risks for coronary artery disease and heart failure.

Keywords: Sleep apnea; obstructive; sleep hypopnea; phenotype; cardiovascular diseases; heart failure

Submitted Aug 09, 2019. Accepted for publication Dec 09, 2019.

doi: $10.21037 /$ jtd.2019.12.66

View this article at: http://dx.doi.org/10.21037/jtd.2019.12.66

\section{Introduction}

Obstructive sleep apnea syndrome (OSAS) is defined as a combination of symptoms and polysomnographic findings that include apneas, hypopneas, and respiratory effortrelated arousal (RERA) (1). OSAS patients have high prevalence rates of obesity, hypertension, and diabetes, all of which are risk factors for cardiovascular disease, and hypoxemia during sleep is also closely related to cardiovascular disease (2). Furthermore, OSAS is associated with other cardiovascular issues, such as coronary artery disease, atrial fibrillation, congestive heart failure, and resistant hypertension, and it has been observed that the 


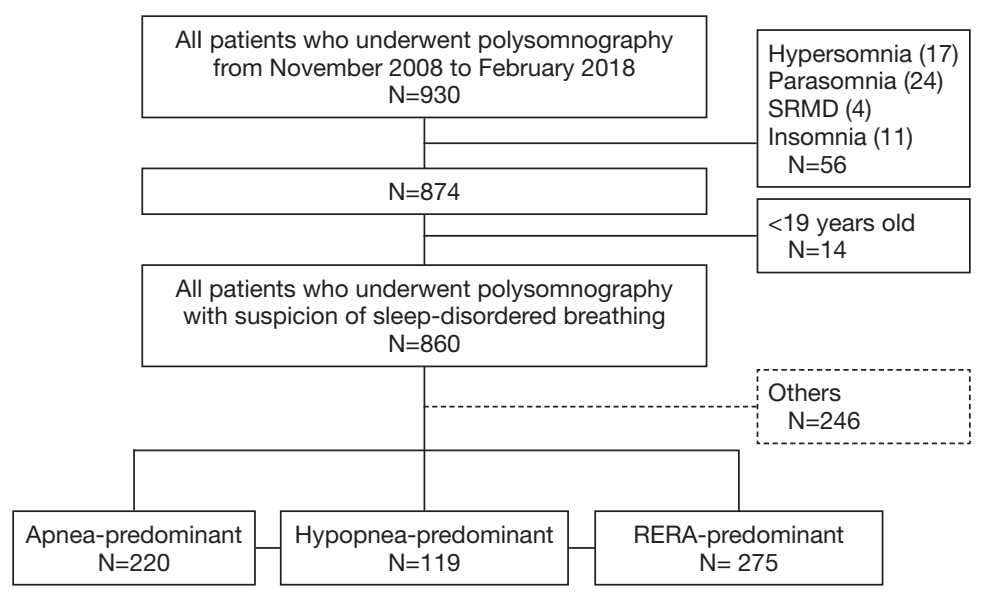

Figure 1 A flow diagram showing the analysis of patients with suspicion of SDB who underwent polysomnography to determine the risk factors for clinical features and cardiovascular disease. The patients were classified as apnea-predominant if apnea accounted for $>50 \%$ of the RDI, hypopnea-predominant if hypopnea accounted for $>50 \%$ of the RDI, and RERA-predominant if RERA accounted for $>50 \%$ of the RDI. SRMD, sleep-related movement disorder; SDB, sleep-disordered breathing; RDI, respiratory disturbance index; RERA, respiratoryeffort related arousal.

prevalence of OSAS increases with the severity of these diseases (3).

It was recently suggested that OSAS is a heterogeneous disease with different clinical features, risk factors, and pathogeneses that depend on the specific phenotype, and as a result, various therapeutic approaches for treating OSAS are being considered (4). Previous studies have reported that the apnea-hypopnea index (AHI) during rapid eye movement (REM) sleep is independently associated with incidents of non-dipping of blood pressure and dyslipidemia (5). Severe sleep apnea during REM sleep may be associated with early signs of atherosclerosis, defined as increased intima thickness (6).

However, few studies have investigated the clinical features of OSAS using phenotypes based on polysomnographic findings.

This study assessed OSAS patients who were classified as apnea-predominant, hypopnea-predominant, or RERApredominant using polysomnography. We evaluated the clinical features of each polysomnographic phenotype and hypothesized that the specific phenotypes would show distinguishable associations with risk for cardiovascular disease.

\section{Methods}

\section{Study setting and participants}

This study assessed patients who were 18 years of age or older, evaluated due to suspicion of sleep-disordered breathing (SDB), and had undergone overnight level 1 polysomnography in the sleep Laboratory of Wonju Severance Christian Hospital (tertiary university hospital) between November 2008 and February 2018 (Figure 1). Polysomnography was conducted in 930 patients, of whom 14 were younger than 18 years of age and were excluded from the study and 56 were patients with indications other than SDB (17 cases of hypersomnia, 24 cases of parasomnia, four cases of sleep-related movement disorders, and 11 cases of insomnia). Thus, a total of 860 SDB patients were included in the final analyses. All procedures performed in studies involving human participants were in accordance with the ethical standards of the Wonju Severance Christian Hospital Institutional Review Board (CR318006).

\section{Sleep study}

All patients underwent all-night in-laboratory 
polysomnography according to standard procedures (7) using a digital system $\left(\right.$ Embla $^{\circledR}$ S7000 \& N7000; Embla Systems Ltd., Kanata, ON, Canada). Based on these data, apnea was considered present if nasal airflow was reduced by more than $90 \%$ of the basal level for more than $10 \mathrm{~s}$; hypopnea was considered present if the nasal pressure fell by more than $30 \%$ of the basal level for more than $10 \mathrm{~s}$, accompanied by a reduction in oxygen saturation of more than $3 \%$ or an arousal (8); and RERA was defined as arousal without apnea or hypopnea. The apnea index, hypopnea index, and RERA index were defined using incidence rate per hour, AHI was calculated as the sum of the apnea and hypopnea rates per hour, and the RDI value was obtained by adding the RERA index to the AHI.

\section{Definition of phenotypes and classification of patient groups}

The OSAS phenotypes were defined and classified using polysomnographic data as follows: Apnea-predominant SDB was defined as when apnea accounted for $>50 \%$ of the RDI; hypopnea-predominant SDB was defined as when hypopnea accounted for $>50 \%$ of the RDI; and RERApredominant SDB was defined as when RERA accounted for $>50 \%$ of the RDI.

\section{Collection of clinical information and risk factors for cardiovascular diseases}

The relevant clinical data of all patients were collected during a review of electronic medical records and included clinical features and sleep-related self-reported questionnaires that were evaluated prior to the sleep study. Demographic information included age, height, weight, body mass index (BMI), and smoking history, and medical history information included comorbidity with diabetes, hypertension, hyperlipidemia, heart failure, and/ or coronary artery disease. In the case of coronary artery disease, relevant data were only included if a patient was hospitalized for myocardial infarction or unstable angina or if they underwent unplanned coronary angiography, cardiovascular angiocardiography, or computed tomography imaging.

\section{Statistical analysis}

Differences in demographic variables according to OSAS phenotype, medical history, and characteristics of the polysomnographic findings are expressed as the mean \pm standard deviation (SD) for continuous variables. Chisquare tests were used to assess nominal variables, and analysis of variance was conducted for comparative analyses of the three groups. Variables that were significantly related to the prevalence of a comorbidity were assessed with logistic regression analyses to estimate the odds ratio (OR) for comorbidity with cardiovascular and cerebrovascular diseases. Significant independent variables were confirmed with multivariate analyses adjusted for other variables. All statistical analyses were performed using SPSS version 24.0, and $\mathrm{P}<0.05$ was considered statistically significant.

\section{Results}

\section{Clinical features according to OSAS phenotype}

The phenotypes of 860 patients were identified based on their polysomnographic data. They were divided into an apnea-predominant group $(\mathrm{n}=220)$, a hypopneapredominant group $(\mathrm{n}=119)$, and a RERA-predominant group ( $\mathrm{n}=275$ ); the remaining 246 patients included 231 individuals who did not fit the characteristics of these three groups and 15 patients with an RDI $<5$ who were excluded from this analysis. The hypopnea-predominant group had a higher mean age $(54.5$ years old, $\mathrm{P}<0.001)$ and higher prevalence rates of hyperlipidemia $(37.6 \%, \mathrm{P}<0.001)$, heart failure $(15.5 \%, \mathrm{P}<0.001)$, and coronary artery disease $(20.9 \%, \mathrm{P}=0.005)$ than the other two groups. The apneapredominant and hypopnea-predominant groups had higher mean prevalence rates of hypertension and diabetes than the RERA-predominant group, and the hypopneapredominant group had a higher ratio of females than the apnea-predominant group. The oxygen desaturation index (ODI) and Epworth Sleepiness Scale (ESS) score were higher in the apnea-predominant group than in the other two groups (Table 1).

\section{Polysomnographic factors for coronary artery diseases and beart failure as risk}

Analyses of the clinical features of the different OSAS phenotypes revealed that the hypopnea-predominant group had higher prevalence rates of coronary artery disease and heart failure than the apnea-predominant group. To investigate polysomnographic data (apnea index, hypopnea index, and RERA index) as risk factors for coronary artery disease and heart failure, simple regression analysis was 
Table 1 Clinical features of the apnea-predominant, hypopnea-predominant, and RERA-predominant groups classified using the polysomnographic data

\begin{tabular}{|c|c|c|c|c|}
\hline Variables & $\begin{array}{l}\text { Apnea-predominant group } \\
\qquad(\mathrm{n}=220)\end{array}$ & $\begin{array}{l}\text { Hypopnea-predominant } \\
\text { group }(n=119)\end{array}$ & $\begin{array}{l}\text { RERA-predominant group } \\
\qquad(\mathrm{n}=275)\end{array}$ & $P$ value \\
\hline Age, years & $48.5 \pm 12.6^{a}$ & $54.5 \pm 14.9^{b}$ & $46.3 \pm 15.8^{a}$ & 0.002 \\
\hline Epworth Sleepiness Scale & $13.7 \pm 2.5^{\mathrm{b}}$ & $11.8 \pm 3.3^{\mathrm{a}}$ & $10.7 \pm 3.3^{a}$ & 0.012 \\
\hline \multicolumn{5}{|l|}{ Anthropometric data } \\
\hline Weight, kg & $79.2 \pm 13.6^{b}$ & $75.2 \pm 15.3^{b}$ & $68.8 \pm 11.6^{a}$ & $<0.001$ \\
\hline Body mass index, $\mathrm{kg} / \mathrm{m}^{2}$ & $27.7 \pm 4.1^{\mathrm{b}}$ & $27.7 \pm 4.9^{b}$ & $24.6 \pm 3.6^{\mathrm{a}}$ & $<0.001$ \\
\hline Neck circumference, $\mathrm{cm}$ & $38.1 \pm 4.9^{b}$ & $36.9 \pm 3.9^{\mathrm{a}}$ & $36.7 \pm 5.4^{a}$ & 0.019 \\
\hline Current smoker, (\%) & $47(22.7)^{\mathrm{b}}$ & $15(13.6)^{\mathrm{a}}$ & $28(10.4)^{a}$ & 0.001 \\
\hline Quantity, pack years & $14.4 \pm 18.4^{b}$ & $10.2 \pm 17.8^{b}$ & $8.1 \pm 15.4^{a}$ & $<0.001$ \\
\hline \multicolumn{5}{|l|}{ Comorbidities } \\
\hline Hypertension, (\%) & $109(52.4)^{b}$ & $63(56.3)^{b}$ & $90(33.5)^{a}$ & $<0.001$ \\
\hline Diabetes, (\%) & $46(22.3)^{b}$ & $25(22.7)^{\mathrm{b}}$ & $26(9.7)^{a}$ & $<0.001$ \\
\hline Hyperlipidemia, (\%) & $51(24.8)^{a}$ & $41(37.6)^{b}$ & $41(15.3)^{\mathrm{a}}$ & $<0.001$ \\
\hline Heart failure, (\%) & $10(4.9)^{\mathrm{a}}$ & $17(15.5)^{\mathrm{b}}$ & $9(3.4)^{\mathrm{a}}$ & $<0.001$ \\
\hline Apnea index & $44.4 \pm 21.9^{b}$ & $6.6 \pm 7.0^{\mathrm{a}}$ & $1.7 \pm 2.5^{\mathrm{a}}$ & $<0.001$ \\
\hline Hypopnea index & $10.0 \pm 8.3^{a}$ & $24.5 \pm 15.7^{b}$ & $3.2 \pm 3.3^{\mathrm{a}}$ & $<0.001$ \\
\hline RERA index & $3.7 \pm 3.7^{\mathrm{a}}$ & $6.9 \pm 4.0^{\mathrm{a}}$ & $12.9 \pm 6.1^{b}$ & $<0.001$ \\
\hline Oxygen desaturation index & $48.4 \pm 23.6^{c}$ & $29.6 \pm 20.3^{b}$ & $4.6 \pm 5.1^{\mathrm{a}}$ & $<0.001$ \\
\hline T90 (min) & $24.5 \pm 36.2^{b}$ & $18.8 \pm 49.4^{\mathrm{b}}$ & $2.1 \pm 16.6^{a}$ & $<0.001$ \\
\hline
\end{tabular}

$\stackrel{a}{\mathrm{~b}, \mathrm{c},}$, values in a row without a common superscript letter differ $(\mathrm{P}<0.05)$, as analyzed by one-way ANOVA. RERA, respiratory-effort related arousal; T90, total sleep time in oxygen saturation $\leq 90 \%$. 
Table 2 Univariate analysis assessing risk factors for coronary artery diseases and heart failure among the patient groups

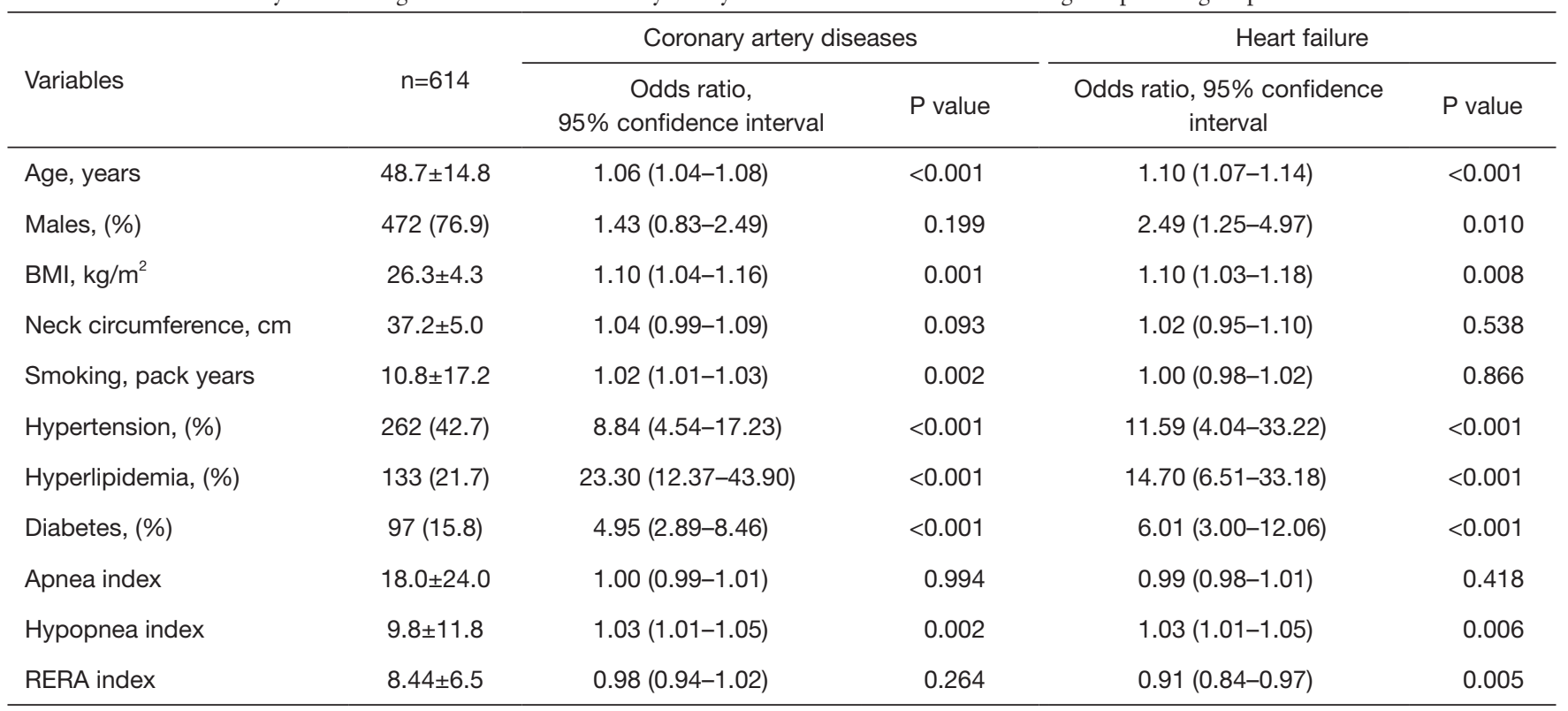

BMI, body mass index; RERA, respiratory effort-related arousal; 95\% CI, 95\% confidence interval.

Table 3 Logistic regression model predicting the risk for coronary artery disease based on severity of the hypopnea index

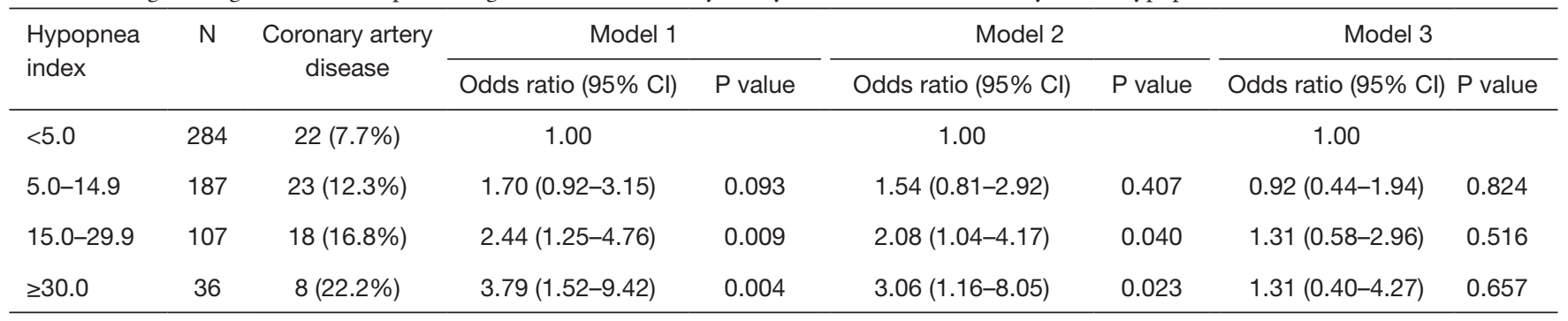

Model 1: hypopnea index. Model 2: hypopnea index, age, sex, and smoking (pack years). Model 3: hypopnea index, age, sex, smoking (pack years), BMI, hypertension, hyperlipidemia, and diabetes. 95\% Cl, 95\% confidence interval.

conducted with these indices including age, sex, BMI, neck circumference, smoking history, presence of hypertension, hyperlipidemia, and diabetes. Age, BMI, hypertension, hyperlipidemia, and diabetes were significantly related to coronary artery disease and heart failure, and of the polysomnographic data, the hypopnea index was a significantly related to heart failure (Table 2).

\section{Coronary artery disease and beart failure according to severity of the bypopnea index}

To determine whether the hypopnea index was independently correlated with coronary artery disease and heart failure, the patients were divided into four groups based on the hypopnea index as follows: $<5,5-14.9$, $15-29.9$, and $\geq 30$. Then, multivariate logistic regression analyses according to severity of the hypopnea index were conducted.

Risk for coronary artery diseases according to the hypopnea index (Table 3)

The group with a hypopnea index from 15 to 29.9 had a 2.44-fold higher risk for coronary artery disease than the group with a hypopnea index $<5$, while the group with a hypopnea index $\geq 30$ had a 3.79-fold higher risk for coronary artery disease than the group with a hypopnea index $<5$. Even when adjusting for age, sex, and smoking history (pack years), these two groups showed significantly higher 
Table 4 Logistic regression model predicting the risk of heart failure based on the severity of the hypopnea index

\begin{tabular}{|c|c|c|c|c|c|c|c|c|}
\hline $\begin{array}{l}\text { Hypopnea } \\
\text { index }\end{array}$ & $\mathrm{n}$ & Heart failure & \multicolumn{2}{|l|}{ Model 1} & \multicolumn{2}{|l|}{ Model 2} & \multicolumn{2}{|l|}{ Model 3} \\
\hline$<5.0$ & 284 & $8(2.8 \%)$ & 1.00 & & 1.00 & & 1.00 & \\
\hline $5.0-14.9$ & 187 & 17 (9.1\%) & 3.51 (1.48-8.31) & 0.004 & $3.22(1.29-8.00)$ & 0.012 & $2.39(0.86-6.60)$ & 0.094 \\
\hline $15.0-29.9$ & 107 & $5(4.7 \%)$ & $1.70(0.54-5.33)$ & 0.362 & $1.42(0.44-4.61)$ & 0.564 & $0.74(0.19-2.88)$ & 0.668 \\
\hline
\end{tabular}

Model 1: hypopnea index. Model 2: hypopnea index, age, sex, and smoking (pack years). Model 3: hypopnea index, age, sex, smoking (pack years), BMI, hypertension, hyperlipidemia, and diabetes. 95\% Cl, 95\% confidence interval.

risks (2.08 and 3.06 times, respectively) than the group with a hypopnea index $<5$. However, after adjusting for BMI and comorbidity with hyperlipidemia and diabetes, these associations were no longer statistically significant.

\section{Risk for heart failure according to the hypopnea index (Table 4)}

The group with a hypopnea index from 5 to 14.9 had a 3.51-fold higher risk for heart failure than the group with a hypopnea index $<5$, whereas the group with a hypopnea index $\geq 30$ had a 7.62 -fold higher risk for heart failure than the group with a hypopnea index $<5$. After adjusting for age, sex, and smoking (pack years), these two groups had significantly higher risks (3.22- and 5.29-fold, respectively) than the group with a hypopnea index $<5$. However, after adjusting for BMI and comorbidity with hyperlipidemia and diabetes, these associations were no longer statistically significant.

\section{Discussion}

This study investigated whether specific phenotypes could show differential associations with risk for cardiovascular diseases in OSAS patients classified into apnea-predominant, hypopnea-predominant, and RERApredominant phenotypes according to polysomnographic data. Individuals with hypopnea-predominant OSAS had higher risks for coronary artery disease and heart failure than those with apnea-predominant or RERA-predominant OSAS.

OSAS is independently associated with metabolic syndrome, which is closely related to cardiovascular diseases (9). OSA is also an independent risk factor for coronary artery calcification, an important factor in predicting cardiovascular disease (10). In addition, it has been reported that patients requiring interventions to treat coronary artery disease were at higher risks of death, myocardial infarction, stroke, repeat revascularization, or heart failure (11).

Almost all previous studies have analyzed the effects of OSAS on cardiovascular and cerebrovascular diseases by classifying severity using the AHI. It has been reported that a higher AHI increases the incidence of cardiovascular diseases (12) and ischemic cerebrovascular disease (13). However, no study has yet investigated the rates of such diseases in patients divided into different groups based on the predominance of either apnea or hypopnea, or both. Therefore, we divided patients into three groups according to polysomnographic phenotype and examined the prevalence and risk factors of cardiovascular disease. The results indicated that hypopnea index was a more important risk factor for coronary artery disease and heart failure than apnea index.

A previous study exploring the clinical significance of hypopnea-predominant and apnea-predominant disorders found that OSAS patients with a BMI of $\geq 45$ had a higher hypopnea index/apnea index ratio than those with a BMI of $<35$ (14). Another study in patients with OSAS accompanied by daytime hypersomnolence found using polysomnographic data that hypopnea constituted $70 \%$ of the RDI in females and 50\% in males (15). Thus, if a patient has a high BMI or is female, hypopnea is more likely to be dominant than apnea. However, we found no BMI difference between the apnea-predominant and hypopneapredominant groups, but observed that the hypopneapredominant group $(38.7 \%)$ had a significantly higher female:male ratio than the apnea-predominant group (7.7\%).

The OSAS pathophysiological phenotypes are: (I) high passive critical closing pressure of the upper airway, (II) 
inadequate responsiveness of the upper airway dilator muscles during sleep, (III) a low respiratory arousal threshold, and (IV) high-loop gain of the respiratory control system (16). Of these, patients with three non-anatomical pathogeneses other than (I) accounted for $50 \%$ of all OSAS patients (17). Non-anatomical pathogeneses trigger dynamic obstruction, while anatomical pathogenesis triggers static obstruction; the pathogeneses were independent and different (18). However, more importantly, a high passive critical closing pressure of the upper airway, an anatomical pathogenesis featuring static obstruction, causes apnea to predominate, whereas the dynamic obstruction caused by non-anatomical pathogeneses mainly causes hypopnea (19). Therefore, apnea and hypopnea are not distinguished simply by the degree of closure, but rather are independent clinical entities featuring different pathogeneses.

The correlation between OSAS and cardiovascular disease is attributable to intermittent hypoxia. When affected patients sleep, breathing disorders occur from 5 to over 100 times per hour, triggering intermittent hypoxia and reoxygenation; the hypoxia causes carotid artery chemoreceptors to activate the sympathetic nerves, in turn increasing hypertension (20). The sympathetic nerve excitation continues during the day, because of a memory effect (plasticity). Recent studies have reported that this effect is a specific response to intermittent hypoxia (21).

Also, oxidative stress caused by hypoxia can trigger systemic inflammation and vascular endothelial cell dysfunction, in turn causing ischemic heart disease, such as myocardial infarction, by inducing atherosclerotic changes in blood vessels $(21,22)$. An excessive increase in intrathoracic pressure imposes mechanical stress on the walls of the large blood vessels of the heart, or creates aortic aneurysms, which in turn trigger arrhythmia (23). Notably, another study on the effect of OSAS on cerebrovascular disease confirmed that reduced middle cerebral artery blood flow correlated with decreased oxygen saturation, and a longer duration thereof, reflecting obstructive hypopnea rather than obstructive apnea or central apnea (24). This may be why obstructive hypopnea reduces cerebral blood flow more than obstructive apnea; the intermittent hypoxia of hypopnea is more severe than that of apnea.

However, ODI, which is an indicator of intermittent hypoxia (25), was higher in the apnea-predominant group than the hypopnea-predominant group. This suggests that the intermittent hypoxia associated with cardiovascular disease cannot be well explained by ODI, and that other tools, such as hypoxia burden, are needed (26).

A limitation of our study is that it was a retrospective analysis of only patients undergoing polysomnography at a single institute. Also, patient numbers were small, and it is thus difficult to define cause-and-effect relationships between OSAS and cardiovascular disease. We did not investigate the hypoxic burden, also known as the desaturation severity parameter, which has been reported to independently raise cardiovascular risk (26). In addition, we did not investigate sleep-related breathing cessation and desaturation, which are known to have greater contributions to daytime sleepiness than AHI (27).

All-cause mortality risk has been reported to be higher in older adult OSA patients with excessive daytime sleepiness (28). In one study, when divided into four subtypes of symptoms, the prevalence of heart failure was more than three times higher in the excessive sleep subtype than other subtypes and the incidence of cardiovascular disease was also high (29). It is necessary to perform followup studies on the factors affecting cardiovascular disease in hypopnea itself because the apnea group had a significantly higher ESS score than the hypopnea group in the present study. It is also necessary to analyze OSA patients by dividing them into subgroups according to ESS score in follow-up studies.

Future studies should divide patients according to polysomnographic phenotype, check various parameters, and then track the incidences of cardiovascular and cerebrovascular diseases, which would identify risk factors and predict prognoses after treatment.

In conclusion, OSAS patients were classified as apnea-predominant, hypopnea-predominant, or RERApredominant according to the phenotype based on polysomnographic data. The hypopnea-predominant group had significantly higher prevalence rates of coronary artery disease and heart failure than the apnea-predominant group. Furthermore, the hypopnea predominance was differentially associated with the risks for coronary artery disease and heart failure.

\section{Acknowledgments}

Funding: None.

\section{Footnote}

Conflicts of Interest: The authors have no conflicts of interest to declare. 
Ethical Statement: The authors are accountable for all aspects of the work in ensuring that questions related to the accuracy or integrity of any part of the work are appropriately investigated and resolved. The study was reviewed and approved by the Wonju Severance Christian Hospital Institutional Review Board (CR318006).

Open Access Statement: This is an Open Access article distributed in accordance with the Creative Commons Attribution-NonCommercial-NoDerivs 4.0 International License (CC BY-NC-ND 4.0), which permits the noncommercial replication and distribution of the article with the strict proviso that no changes or edits are made and the original work is properly cited (including links to both the formal publication through the relevant DOI and the license). See: https://creativecommons.org/licenses/by-nc-nd/4.0/.

\section{References}

1. Shin C. Epidemiology and definition of sleep disordered breathing. Tuberc Respir Dis 2009;66:1-5.

2. Stoohs R, Guilleminault C. Cardiovascular changes associated with obstructive sleep apnea syndrome. J Appl Physiol 1992;72:583-9.

3. McEvoy RD, Antic NA, Heeley E, et al. CPAP for prevention of cardiovascular events in obstructive sleep apnea. N Engl J Med 2016;375:919-31.

4. Zinchuk AV, Gentry MJ, Concato J, et al. Phenotypes in obstructive sleep apnea: A definition, examples and evolution of approaches. Sleep Med Rev 2017;35:113-23.

5. Mokhlesi B, Finn LA, Hagen EW, et al. Obstructive sleep apnea during REM sleep and hypertension. results of the Wisconsin Sleep Cohort. Am J Respir Crit Care Med 2014;190:1158-67.

6. Ljunggren M, Lindberg E, Franklin KA, et al. Obstructive sleep apnea during rapid eye movement sleep is associated with early signs of atherosclerosis in women. Sleep 2018. doi: 10.1093/sleep/zsy099.

7. Berry RB, Budhiraja R, Gottlieb DJ, et al. Rules for scoring respiratory events in sleep: Update of the 2007 AASM Manual for the Scoring of Sleep and Associated Events. Deliberations of the Sleep Apnea Definitions Task Force of the American Academy of Sleep Medicine. J Clin Sleep Med 2012;8:597-619.

8. Iber C. The AASM manual for the scoring of sleep and associated events: Rules, terminology and technical specifications. 1st ed. Westchester, IL: American Academy of Sleep Medicine; 2007.
9. Drager LF, Polotsky VY, O'Donnell CP, et al. Translational approaches to understanding metabolic dysfunction and cardiovascular consequences of obstructive sleep apnea. Am J Physiol Heart Circ Physiol 2015;309:H1101-11.

10. Lutsey PL, McClelland RL, Duprez D, et al. Objectively measured sleep characteristics and prevalence of coronary artery calcification: The Multi-Ethnic Study of Atherosclerosis Sleep study. Thorax 2015;70:880-7.

11. Wang X, Fan JY, Zhang Y, et al. Association of obstructive sleep apnea with cardiovascular outcomes after percutaneous coronary intervention: A systematic review and meta-analysis. Medicine (Baltimore) 2018;97:e621.

12. Kendzerska T, Gershon AS, Hawker G, et al. Obstructive sleep apnea and risk of cardiovascular events and all-cause mortality: A decade-long historical cohort study. PLoS Med 2014;11:e1001599.

13. Redline S, Yenokyan G, Gottlieb DJ, et al. Obstructive sleep apnea-hypopnea and incident stroke: The sleep heart health study. Am J Respir Crit Care Med 2010;182:269-77.

14. Mathew R, Castriotta RJ. High hypopnea/apnea ratio (HAR) in extreme obesity. J Clin Sleep Med 2014;10:391-6.

15. Rowley JA, Zhou XS, Diamond MP, et al. The determinants of the apnea threshold during NREM sleep in normal subjects. Sleep 2006;29:95-103.

16. Bosi M, De Vito A, Gobbi R, et al. The importance of obstructive sleep apnoea and hypopnea pathophysiology for customized therapy. Eur Arch Otorhinolaryngol 2017;274:1251-61.

17. Eckert DJ, White DP, Jordan AS, et al. Defining phenotypic causes of obstructive sleep apnea. Identification of novel therapeutic targets. Am J Respir Crit Care Med 2013;188:996-1004.

18. Farré R, Rigau J, Montserrat JM, et al. Static and dynamic upper airway obstruction in sleep apnea: Role of the breathing gas properties. Am J Respir Crit Care Med 2003;168:659-63.

19. Montserrat JM, Farré R, Navajas D. New technologies to detect static and dynamic upper airway obstruction during sleep. Sleep Breath 2001;5:193-206.

20. Khayat R, Pleister A. Consequences of obstructive sleep apnea: Cardiovascular risk of obstructive sleep apnea and whether continuous positive airway pressure reduces that risk. Sleep Med Clin 2016;11:273-86.

21. Peng YJ, Prabhakar NR. Reactive oxygen species in the plasticity of respiratory behavior elilcited by chronic intermittent hypoxia. J Appl Physiol 2003;94:2342-9.

22. Dewan NA, Nieto FJ, Somers VK. Intermittent 
hypoxemia and OSA: Implications for comorbidities. Chest 2015;147:266-74.

23. Clarenbach CF, Camen G, Sievi NA, et al. Effect of simulated obstructive hypopnea and apnea on thoracic aortic wall transmural pressures. J Appl Physiol (1985) 2013;115:613-7.

24. Leslie WD, Wali S, Kryger M. Blood flow of the middle cerebral artery with sleep-disordered breathing: Correlation with obstructive hypopneas. Stroke 1999;30:188-90.

25. Fukuoka R, Kohno T, Kohsaka S, et al. Nocturnal intermittent hypoxia and short sleep duration are independently associated with elevated C-reactive protein levels in patients with coronary artery disease. Sleep Med 2017;29:29-34.

26. Azarbarzin A, Sands SA, White DP, et al. The hypoxic

Cite this article as: Park S, Shin B, Lee JH, Lee SJ, Lee MK, Lee WY, Yong SJ, Kim SH. Polysomnographic phenotype as a risk factor for cardiovascular diseases in patients with obstructive sleep apnea syndrome: a retrospective cohort study. J Thorac Dis 2020;12(3):907-915. doi: 10.21037/jtd.2019.12.66 burden: A novel sleep apnoea severity metric and a predictor of cardiovascular mortality-Reply to 'The hypoxic burden: Also known as the desaturation severity parameter.' Eur Heart J 2019;40:2994-5.

27. Kainulainen S, Toyras J, Oksenberg A, et al. Severity of desaturations reflects OSA-related daytime sleepiness better than AHI. J Clin Sleep Med 2019;15:1135-42.

28. Gooneratne NS, Richards KC, Joffe M, et al. Sleep disordered breathing with excessive daytime sleepiness is a risk factor for mortality in older adults. Sleep 2011;34:435-42.

29. Mazzotti DR, Keenan BT, Lim DC, et al. Symptom subtypes of obstructive sleep apnea predict incidence of cardiovascular outcomes. Am J Respir Crit Care Med 2019;200:493-506. 